\title{
Complete heart block in an elderly patient with Polysplenia Syndrome: A case report
}

\author{
Keywords: Complete heart block - Interrupted inferior vena cava - Polysplenia syndrome
}

\section{Introduction}

Polysplenia syndrome was first described by Abernethy in 1793 [1] and a full description was given by Moller et al. [2] in 1967. In left atrial isomerism the most prevalent alterations are: polysplenia, presence of two morphologically left lungs, two morphologically left atrial appendages, and absence of the hepatic segment of the inferior vena cava, midline liver, and high incidence of congenital heart defects.

The congenital heart defects most frequently associated with isomerism of the left atrial appendages are: double right ventricular outflow tract, single atrium, atrioventricular septal defect, pulmonary valve stenosis, and persistence of the left superior vena cava $[3,4]$. Heart rhythm disturbances are also described, most common being a wandering pacemaker $[5,6]$. Another common finding is the decrease in heart rate as age increases. At fifteen years of age, $70 \%$ of the patients present with sinus bradycardia. Atrioventricular block may be present in $50 \%$ of the patients in some phase of their lives and is progressive in nature [7].
Histological studies show the absence of the sinus node in the junction of the superior vena cava with the right atrium [5]. A hypoplastic and abnormally located sinus node has been demonstrated in the upper lateral atrial wall, in the medial lateral atrial wall, and in the lower lateral wall, close to the coronary sinus ostium. In most instances the sinus node cannot be found [8].

\section{Case Report}

A 65-year-old female patient was admitted in our hospital with a history of recurrent syncope of 1 year duration. On enquiry, patient gave history three episodes of syncope over past one year. On examination, she was having pulse rate of 50/ min with regular rhythm. Her blood pressure (BP) was $140 / 90 \mathrm{mmHg}$. Cardiovascular system examination was normal. Investigations revealed a normal complete blood count and blood sugar. Serum electrolytes, kidney function tests and liver function tests were within normal range. Electrocardiography (ECG) showed complete heart block with a junctional escape rhythm at 55 per minute (Figure 1). Patient was

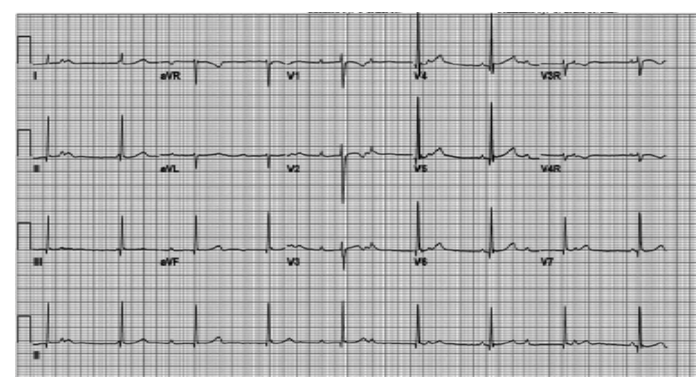

Figure 1: ECG of the patient showing complete heart block. 
taken up for temporary pacemaker insertion. Catheter course was from right femoral vein to inferior vena cava to azygous vein to left superior vena cava to coronary sinus to right atrium to right ventricle. IVC venogram (Video 1), (Figure 2) and azygous venogram (Video 2) revealed infra-hepatic IVC interruption with azygous continuation draining into left SVC. Ultrasonography (USG) showed liver in midline and more towards left side, hilum leftward, but inferior vena cava (IVC) and hepatic veins on right side and were normal. No spleen on left side was identified consistent with situs ambiguous abdominis. Echo showed dilated coronary sinus with left SVC and normal left ventricular ejection fraction. Contrast-enhanced computed tomography (CECT) abdomen was performed on a 128 slice dual source dual energy scanner and it showed evidence of liver, gallbladder, IVC and common bile duct

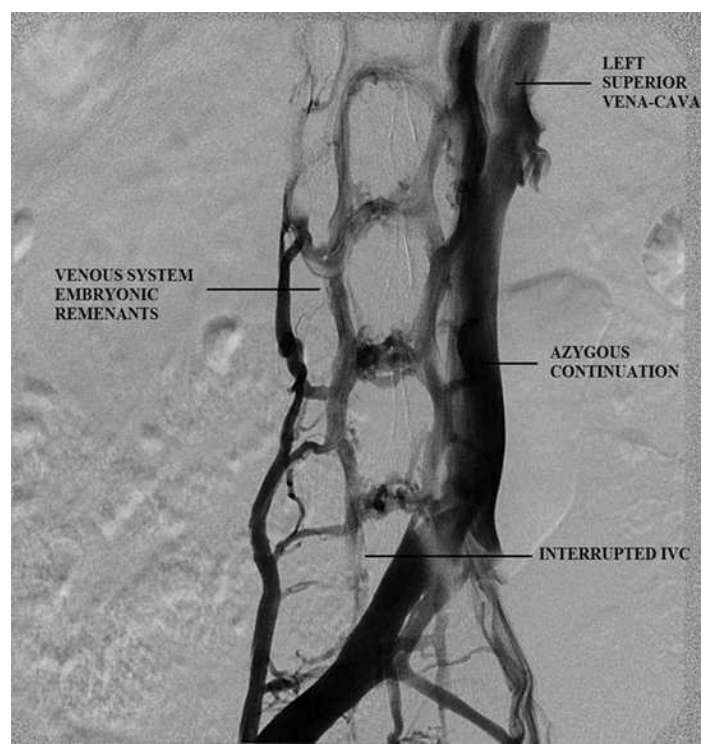

Figure 2: Digital subtraction angiographic image of venous angiogram taken from right femoral vein showing interrupted inferior vena cava continuing as azygous vein which further continues as left superior vena cava draining into the coronary sinus and right atrium.

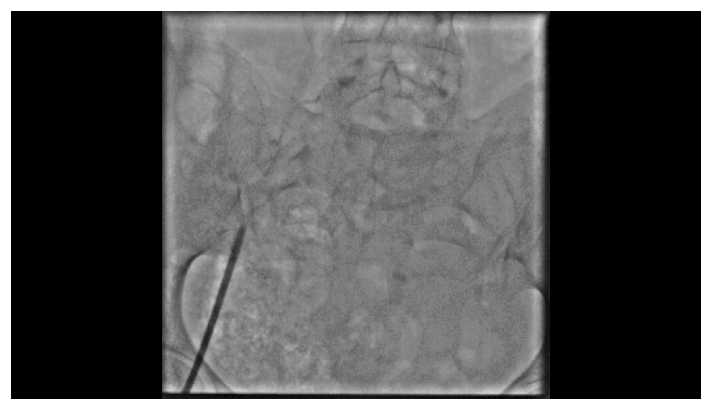

Video 1: Venous angiogram of inferior vena cava. Venous angiogram taken from right femoral vein showing interrupted inferior vena cava continuing as azygous vein which further continues as left superior vena cava draining into the coronary sinus and right atrium.

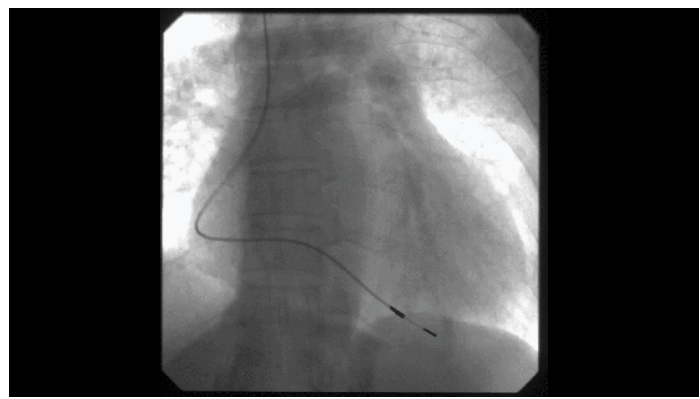

Video 2: Venous angiogram from azygous vein. Venous angiogram of interrupted IVC taken with an injection into azygous vein showing connecting remnant from azygous to the right superior vena cava and continuation of azygous to left superior vena cava. 


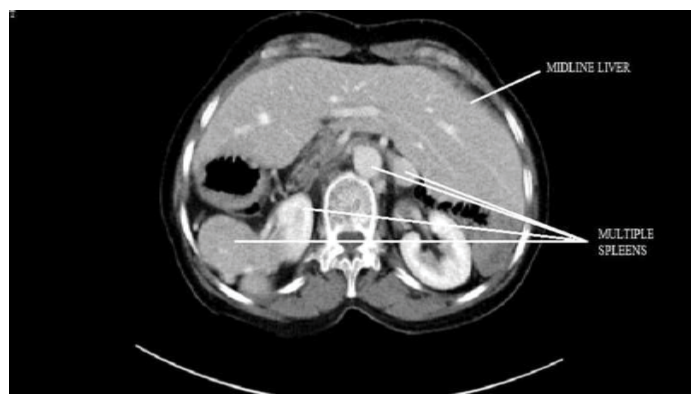

Figure 3: Contrast enhanced CT scan image showing midline liver and multiple accessory spleens in abdomen.

on left side of abdomen (Figure 3). Multiple accessory spleens (at least 7) were seen in the abdomen. A final impression of situs ambigus with polysplenia syndrome was made on CECT abdomen. DDDR pacemaker was implanted in the patient for $\mathrm{CHB}$.

\section{Discussion}

Polysplenia is a complex syndrome with a high variability of anatomical findings. Three types of heart rhythm disturbances may occur in the isomerism of the left atrial appendages: sinus node dysfunction [5], atrioventricular block [3,4], and dual atrioventricular nodal pathways [5]. Various mutations have been identified in patients with Polysplenia syndrome like Z1C3, LEFTYA3 etc. [8]. However, such mutations were not studied or identified in our patient.
The patient reported was an adult diagnosed with polysplenia syndrome with a structurally normal heart and CHB. Patients of polysplenia syndrome with structurally normal heart presenting with isolated conduction disorder is a very unusual and rare occurrence [5]. In patients with $\mathrm{CHB}$ the outcome is better when the AAI C/R and DDD C/R pacemakers are used for electrical stimulation because of the lower prevalence of atrial fibrillation, thromboembolic events, and heart failure [3]. Very few case reports on patients with structurally normal hearts in polysplenia syndrome presenting with $\mathrm{CHB}$ are available. After a four-year follow-up, the patient's outcome remains satisfactory.

\section{Executive summary}

Very few case reports on patients with structurally normal hearts in polysplenia syndrome presenting with $\mathrm{CHB}$ are available. After a four-year follow-up, the patient's outcome remains satisfactory.

\section{References}

1. Abernethy J. Account of two instances of uncommon formation in the viscera of human body. Philos. Trans. R. Soc. Lond. 83: 59-66 (1793).

2. Moller JH, Nakib A, Anderson RC, Edwards JE. Congenital cardiac disease associated with polysplenia: a developmental complex of bilateral left-sidedness. Circulation. 36: 789-799 (1967).

3. Chiu IS, How SW, Wang JK, et al. Clinical implications of atrial isomerism. Br. Heart. J. 60: 72-77 (1988).

4. Fukuda K, Onda T, Kimura Y, et al. An adult case of polysplenia syndrome associated with sinus node dysfunction, dextrocardia, and systemic venous anomalies. Intern. Med. 54(9): 1071-1074 (2015).

5. Momma K, Takao A, Shibata T. Characteristics and natural history of abnormal atrial rhythms in left isomerism. Am. J. Cardiol. 65: 231-236 (1990).

6. Loomba RS, Willes RJ, Kovach JR, Anderson RH. Chronic Arrhythmias in the Setting of Heterotaxy: Differences between Right and Left Isomerism. Congenit. Heart. Dis. 11(1): 7-18 (2016).

7. Wren C, Macartney FJ, Deanfield JE. Cardiac rhythm in atrial isomerism. Am. J. Cardiol. 59: 1156-1158 (1987).

8. Zhu L, Belmont JW, Ware SM. Genetics of human heterotaxias. Eur. J. Hum. Genet. 14(1): 17-25 (2006). 